Case Report

\title{
Low-Grade Malignant Triton Tumor of the Neck: A Case Report and Review of the Literature
}

\author{
Taissir Omar, ${ }^{1}$ Hanaa Raslan,, Sahar El Sheikh, ${ }^{1}$ \\ Moataz Rizq, ${ }^{2}$ and Awatef Draz ${ }^{3,4}$ \\ ${ }^{1}$ Oral Pathology Department, Faculty of Dentistry, Alexandria University, Champollion Street, Azarita, Alexandria 21526, Egypt \\ ${ }^{2}$ Maxillofacial and Plastic Surgery Department, Faculty of Dentistry, Alexandria University, Champollion Street, Azarita, \\ Alexandria 21526, Egypt \\ ${ }^{3}$ Oral Pathology Department, Faculty of Dentistry, King Abdulaziz University, P.O. Box 80200, Zip Code 21589, \\ Jeddah 22252, Saudi Arabia \\ ${ }^{4}$ Cairo University, 12 EL-Saraya Street, Manial, Cairo 11553, Egypt
}

Correspondence should be addressed to Awatef Draz; awatefdraz@yahoo.com

Received 6 July 2014; Accepted 17 August 2014; Published 22 September 2014

Academic Editor: Imtiaz A. Chaudhry

Copyright (c) 2014 Taissir Omar et al. This is an open access article distributed under the Creative Commons Attribution License, which permits unrestricted use, distribution, and reproduction in any medium, provided the original work is properly cited.

Rhabdomyoblastic differentiation in a malignant peripheral nerve sheath tumor (MPNST) is termed malignant triton tumor (MTT), a rare neoplasm that poses a diagnostic dilemma in the differential diagnosis of neck masses and portends poor prognosis. We report a sporadic case of MTT of the neck in a 23-year-old female. We present the pathological findings. Immunohistochemistry confirmed the neurogenic origin with S-100 expression and the rhabdomyoblastic differentiation with desmin and vimentin positivity. Radical surgical excision was done. After 4 years there were no signs of recurrence or distant metastasis. The clinical, microscopic, and long-term follow-up of this case are consistent with those of a low-grade malignancy.

\section{Introduction}

Malignant peripheral nerve sheath tumor (MPNST) is a rare soft tissue neoplasm with a poor prognosis. It can occur in association with neurofibromatosis type 1 (NF-1) or sporadically accounting for $5-10 \%$ of soft tissue sarcomas [14]. MPNST occurs in about $2-5 \%$ of patients with NF-1, compared with a prevalence of $0.001 \%$ in the general population $[5,6]$. MPNST comprises only $2-6 \%$ of head and neck sarcomas [7]. The diagnosis, management, and histogenesis of MPNST continue to challenge pathologists and surgeons especially in sporadic cases $[3,8]$. These tumors are thought to arise from Schwann cells, their precursors, or a preexisting NF-1 [4]. MPNST may consist of tissues such as glandular epithelium, squamous cells, cartilage, bone, or even adipose tissue. Tumors with skeletal muscle differentiation and malignant Schwann cells are classified as a subtype or a histologic variant of MPNST $[9,10]$ and are referred to as malignant triton tumor (MTT) [11]. This tumor was originally described by Masson in 1932. It is very rare and has a more aggressive clinical course and worse prognosis than MPNST without rhabdomyoblastic differentiation [12-14]. It was reported that the 5 -year survival rate is $5-15 \%[13,14]$, compared to $50-60 \%$ for MPNST [15].

In the majority of reported cases, triton tumors are located across peripheral nerves, usually close to the spine, in the head and neck region, or in the upper and lower extremities [16]. However, an intracardiac presentation has been reported within the atrium [17]. Wong et al. [18] reported that MTT involving nonextremity sites has a worse prognosis. In fact, the location, large size, and tumor stage are found to affect survival $[15,18]$. The metastatic rate in patients with MTT is reported to be $31.4 \%$ [13]. Shorter survival was reported in cases of MTT not associated with NF-1 [11]. 


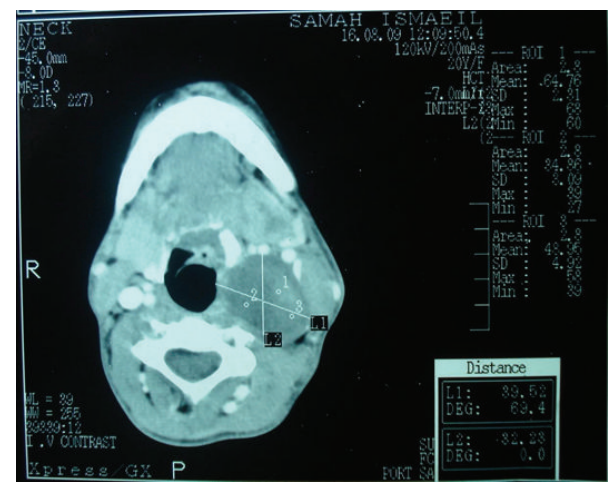

Figure 1: Axial view of CT showing dimensions of the tumor.

Two theories were proposed as an explanation to the development of this tumor [19]. The first was that Schwann cells in neurogenic tumor could be stimulated by motor nerve to differentiate into rhabdomyoblastic component. The second explanation was more plausible, where neoplastic Schwann cells can transform into rhabdomyoblasts, suggesting the possibility of some mesenchymal tissue derivation from neuroectodermal cells. Another opinion suggested a differentiation-metaplasia capacity of neuroectodermal tissue $[20]$.

To further elucidate the natural history and prognosis of this rare neoplasm in the head and neck, we present a case of sporadic MTT arising in the neck with an unusual prognosis.

\section{Case Presentation}

A 23-year-old female patient presented to the craniomaxillofacial and plastic surgery department at our institution with a 10-month left neck swelling. Her medical and family histories were unremarkable and negative for prior radiation exposure and NF-1, respectively. Physical examination revealed a slightly tender, firm mass, fixed to the underlying structures, with a normal overlying skin and no palpable lymphadenopathy.

Conventional computed tomography (CT) (Figure 1) and CT angiography (Figure 2) demonstrated a left parapharyngeal mass $3 \times 4 \times 9 \mathrm{~cm}$, displacing the left internal carotid artery, external carotid artery, and internal jugular vein, with no evidence of vascular infiltration. An incisional biopsy revealed the diagnosis of sporadic-type MTT (de novo) of the neck. This was followed by a wide surgical excision of the mass under general anesthesia.

Macroscopically, the tumor was oval in shape, partially encapsulated with a smooth yellowish-white surface. The cut surface showed microcysts, hemorrhage, and necrosis with brownish areas (Figure 3). On microscopic examination. A partially encapsulated cellular mass composed of interlacing fascicles of hyperchromatic serpentine spindle cells was found. The cells had elongated and comma shaped nuclei with prominent nucleoli and sparse cytoplasm with indistinct borders. Large pleomorphic cells with abundant eosinophilic cytoplasm and rounded eccentric nuclei with large nucleoli were present (Figures 4(a), 4(b), and 4(c)). Some of these

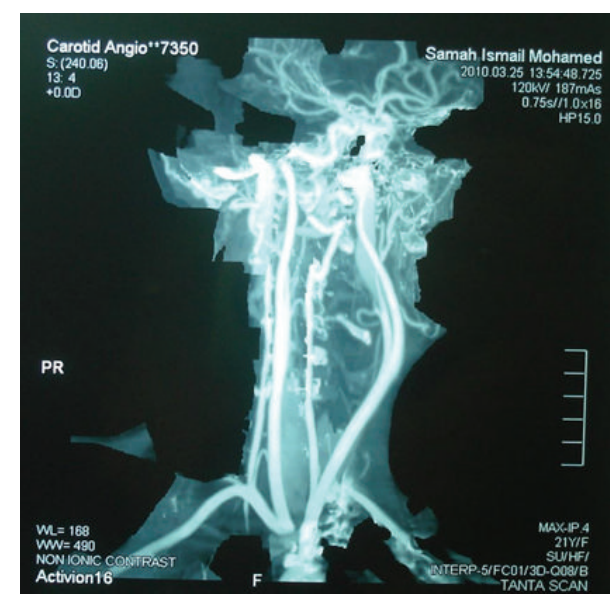

FIGURE 2: Coronal view of CT angiography showing left parapharyngeal and pharyngeal mainly hypodense, peripherally hyperdense well-defined lesion $(7 \times 4 \times 3 \mathrm{~cm})$ indenting the left pharyngeal wall but not infiltrating it.

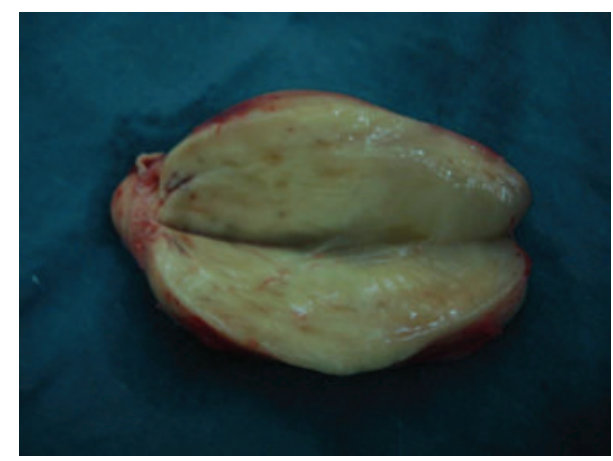

FIGURE 3: Cross-sectional image of the excised tumor.

identified cells were striated, strap-like, or globoid rhabdomyoblasts. These cells were identified on hematoxylin and eosin and phosphotungstic acid hematoxylin stains (Figure 4(d)). Necrosis was limited to few areas. The surgical margins were free of disease. Immunohistochemical staining (IHS) of the tumor revealed diffuse, intense positivity of the wavy spindle cells for S-100 protein, confirming its neurogenic origin (Figure 5(a)). Myogenic differentiation of the large pleomorphic cells was confirmed by the strong positivity for desmin (Figure 5(b)) and moderate positivity for myoglobin (Figure 5(c)). The microscopic examination and the reaction to the previous markers confirmed the diagnosis of a low grade MTT.

The annual follow-up CT scans revealed no evidence of recurrence or metastases. Our patient is alive and still free of recurrences four years after initial diagnosis.

\section{Discussion}

A so-called mosaic tumor with both muscular and neurogenic components. In spite of being a rare tumor, the head and neck region is one of the most frequent sites of involvement of MTT. For this reason, head and neck surgeons and 


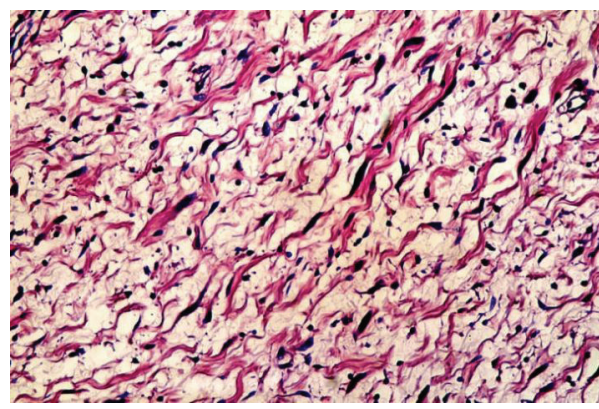

(a)

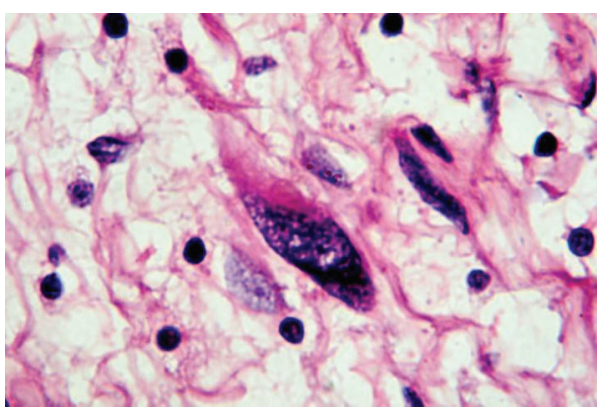

(c)

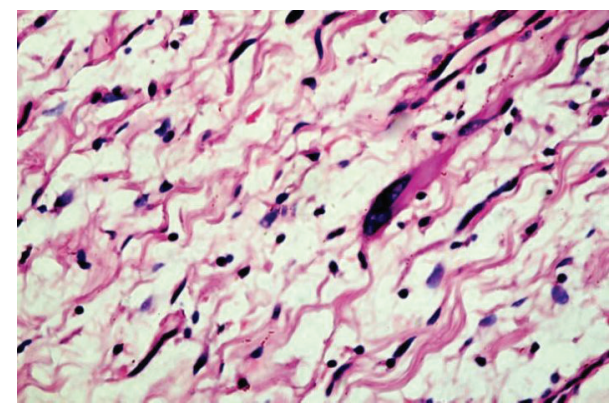

(b)

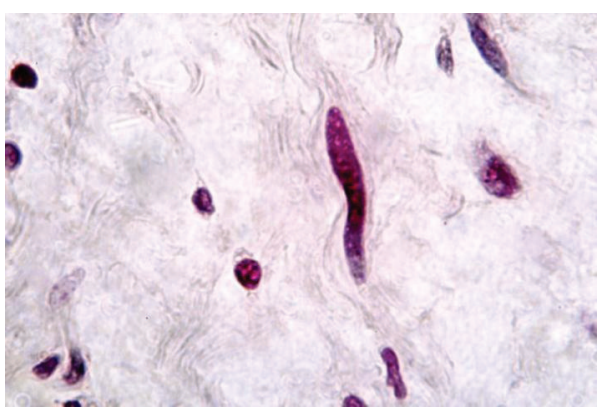

(d)

FIGURE 4: Malignant triton tumor showing the following: (a) well delineated spindle cells with scattered rhabdomyoblasts (H\&E $\times 100$ ), $((b)$ and $(c))$ higher magnification $(\times 200$ and $\times 400)$, and $(d)$ cross striations of rhabdomyoblast cells stained with phosphotungstic acid hematoxylin $(\times 400)$.

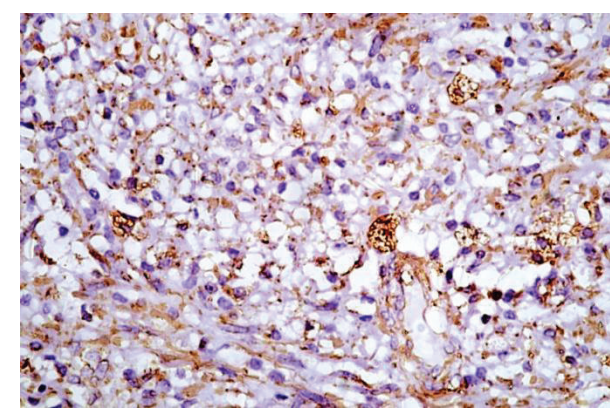

(a)

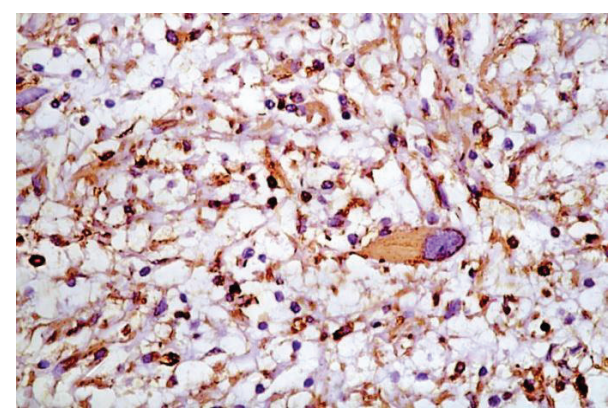

(b)

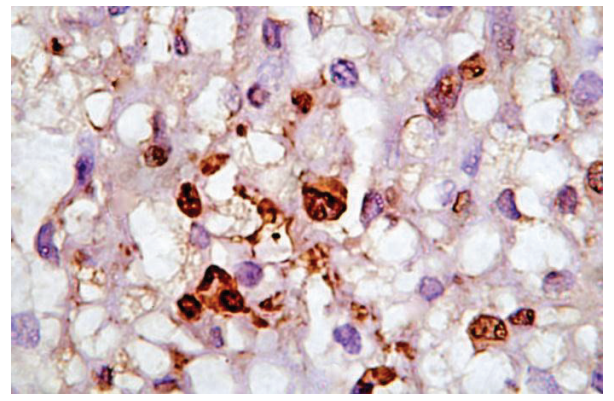

(c)

FIGURE 5: Immunohistochemical staining of malignant triton tumor showing the following: (a) diffuse positivity for S-100 protein $(\times 200)$, (b) desmin positivity in the rhabdomyoblast cells $(\times 200)$, and (c) myoglobin positivity in the rhabdomyoblast cells $(\times 400)$. 
pathologists should be familiar with the nature, the treatment modalities, and prognosis of this rare tumor [21, 22].

Our reported case in the neck was sporadic. It seems that the head and neck neoplasms are infrequently associated with NF-1 compared to those located in other sites [22]. Our patient's age falls within the average age of 30 years reported in the literature [23]. However, a wide age range from newborns to extremely older individuals has been reported by others $[24,25]$.

In the present case, the IHS was essential to reach the proper diagnosis. Tumor morphology of a malignant schwannoma with potentially overlooking the neoplastic rhabdomyoblasts makes IHS essential in the diagnosis of MTT. However, Weiss and Enzinger [26] mentioned that discrete rhabdomyoblastic differentiation is necessary to label a MTT. The considerable difficulty to diagnose this tumor is attributed to its microscopic similarity to a wide variety of sarcomas [27]. Therefore, IHS is important. This is in accordance with other investigators [23, 27-29]. Furthermore, it was found that electron microscopic study can be used in the diagnosis of these tumors, although not performed in the present case $[3,27,29]$.

Once the diagnosis was done, complete tumor resection with negative margins should be the goal of surgery, as in the present case. Therefore, it would certainly make sense to consider the use of such treatment without delay. This is consistent with other researchers [21, 30, 31].

The visibility of the neck mass in our reported case made its timely diagnosis possible. Reports of tumors in noticeable sites aid in the it's early diagnosis and excision while the tumor is still small leading to a better prognosis. Such areas include the extremities [18] and the head and neck [21, 30, 31]. This is in contrast to the findings of other investigators [12$14]$.

The size of the tumor is also an important prognostic factor affecting survival. In the current case, the tumor size was smaller than $10 \mathrm{~cm}$. It was mentioned that patients with tumors less than $10 \mathrm{~cm}$ had better survival than those with larger tumors $[3,18,32]$. This could probably be related to the stage of the tumor at the time of presentation.

Victoria et al. [28] reviewed the treatment and outcome of 27 MTTs arising in the head and neck and commented that there may be a subset of MTT occurring in this region as a low-grade malignancy with favorable long-term prognosis. Their findings were supported by other researchers $[30,31]$. Unlike the previous opinions, it was reported that rhabdomyoblastic differentiation within the tumor is fatal and causes it to behave aggressively [2, 4, 12-14].

Furthermore, our case was sporadic and of low-grade malignancy. This is in accordance with Brooks et al. [25] who reported that when MTT is not associated with NF-1, it is usually of low grade.

The clinical, microscopic, and long-term follow-up of the present case showed that our case was a low-grade malignancy. Finally, we would like to emphasize that early diagnosis and wide surgical treatment are important for the prognosis of MTT.

\section{Conflict of Interests}

The authors declare that there is no conflict of interests regarding the publication of this paper.

\section{References}

[1] M. H. Haddadin, A. L. Hawkins, P. Long et al., "Cytogenetic study of malignant triton tumor: a case report," Cancer Genetics and Cytogenetics, vol. 144, no. 2, pp. 100-105, 2003.

[2] C. J. Stasik and O. Tawfik, "Malignant peripheral nerve sheath tumor with rhabdomyosarcomatous differentiation (malignant triton tumor)," Archives of Pathology and Laboratory Medicine, vol. 130, no. 12, pp. 1878-1881, 2006.

[3] J. Pfeiffer, I. Arapakis, C. C. Boedeker, and G. J. Ridder, "Malignant peripheral nerve sheath tumour of the paranasal sinuses and the anterior skull base," Journal of Cranio-Maxillofacial Surgery, vol. 36, no. 5, pp. 293-299, 2008.

[4] R. Prieto, J. M. Pascual, M. A. García-Cabezas, F. López-Barea, L. Barrios, and F. González-Llanos, "Low-grade malignant triton tumor in the lumbar spine: a rare variant of malignant peripheral nerve sheath tumor with rhabdomyoblastic differentiation," Neuropathology, vol. 32, no. 2, pp. 180-189, 2012.

[5] J. M. Baehring, R. A. Betensky, and T. T. Batchelor, "Malignant peripheral nerve sheath tumor: the clinical spectrum and outcome of treatment," Neurology, vol. 61, no. 5, pp. 696-698, 2003.

[6] T. Sabesan, K. Hussein, and V. Ilankovan, "Malignant peripheral nerve sheath tumour of the parapharyngeal space in a patient with neurofibromatosis type 1," British Journal of Oral and Maxillofacial Surgery, vol. 46, no. 7, pp. 585-587, 2008.

[7] T. R. Loree, J. H. North Jr., B. A. Werness, R. Nangia, A. P. Mullins, and W. L. Hicks Jr., "Malignant peripheral nerve sheath tumors of the head and neck: analysis of prognostic factors," Otolaryngology-Head and Neck Surgery, vol. 122, no. 5, pp. 667-672, 2000.

[8] C. Kobayashi, Y. Oda, T. Takahira et al., "Chromosomal aberrations and microsatellite instability of malignant peripheral nerve sheath tumors: a study of 10 tumors from nine patients," Cancer Genetics and Cytogenetics, vol. 165, no. 2, pp. 98-105, 2006.

[9] E. N. McComb, R. D. McComb, J. M. DeBoer, J. R. Neff, and J. A. Bridge, "Cytogenetic analysis of a malignant triton tumor and a malignant peripheral nerve sheath tumor and a review of the literature," Cancer Genetics and Cytogenetics, vol. 91, no. 1, pp. 8-12, 1996.

[10] C. Osyo, I. Aslan, B. Biglic, and E. Yazicioglu, "Malignant triton tumor of the parapharyngeal space," Journal of Laryngology and Otology, vol. 115, pp. 573-575, 2001.

[11] E. Aldlyami, A. Dramis, R. J. Grimer, A. Abudu, S. R. Carter, and R. M. Tillman, "Malignant triton tumour of the thigh-a retrospective analysis of nine cases," European Journal of Surgical Oncology, vol. 32, no. 7, pp. 808-810, 2006.

[12] K. Tripathy, R. Mallik, A. Mishra et al., "A rare malignant triton tumor," Case Reports in Neurology, vol. 2, no. 2, pp. 69-73, 2010.

[13] Y. J. McConnell and C. A. Giacomantonio, "Malignant triton tumors-complete surgical resection and adjuvant radiotherapy associated with improved survival," Journal of Surgical Oncology, vol. 106, no. 1, pp. 51-56, 2012. 
[14] S. C. Kamran, S. A. Howard, A. B. Shinagare et al., "Malignant peripheral nerve sheath tumors: prognostic impact of rhabdomyoblastic differentiation (malignant triton tumors), neurofibromatosis 1 status and location," European Journal of Surgical Oncology, vol. 39, no. 1, pp. 46-52, 2013.

[15] C.-C. H. Stucky, K. N. Johnson, R. J. Gray et al., "Malignant peripheral nerve sheath tumors (MPNST): the Mayo Clinic experience," Annals of Surgical Oncology, vol. 19, no. 3, pp. 878885, 2012.

[16] C. Zisis, S. Fragoulis, D. Rontogianni, G. Stratakos, and I. Bellenis, "Malignant triton tumour of the anterior mediastinum as incidental finding," Monaldi Archives for Chest Disease, vol. 65, no. 4, pp. 222-224, 2006.

[17] S. Kabir, E. I. Kapetanakis, and F. Shabbo, "Intracardiac malignant Triton tumor: a first presentation," Annals of Thoracic Surgery, vol. 89, no. 3, pp. 968-969, 2010.

[18] W. W. Wong, T. Hirose, B. W. Scheithauer, S. E. Schild, and L. L. Gunderson, "Malignant peripheral nerve sheath tumor: analysis of treatment outcome," International Journal of Radiation Oncology Biology Physics, vol. 42, no. 2, pp. 351-360, 1998.

[19] P. Masson, Recklinghausen's Neurofibromatosis. Sensory Neuromas and Motor Neuromas, Libman Anniversary, vol. 2, International Press, New York, NY, USA, 1932.

[20] B. S. Ducatman and B. W. Scheithauer, "Malignant peripheral nerve sheath tumors with divergent differentiation," Cancer, vol. 54, no. 6, pp. 1049-1057, 1984.

[21] B. Rekhi, N. A. Jambhekar, A. Puri, M. Agrawal, and R. F. Chinoy, "Clinicomorphologic features of a series of 10 cases of malignant triton tumors diagnosed over 10 years at a tertiary cancer hospital in Mumbai, India," Annals of Diagnostic Pathology, vol. 12, no. 2, pp. 90-97, 2008.

[22] L. Barnes, Surgical Pathology of the Head and Neck, vol. 2, 2nd edition, 2009.

[23] E. Özer, S. Erklç, Y. A. Bayazt, S. Mumbuç, A. Aydn, and M. Kanlkama, "Malignant triton tumor of the supraclavicular region arising after radiotherapy," Auris Nasus Larynx, vol. 29, no. 4, pp. 405-407, 2002.

[24] Y. Daimaru, H. Hashimoto, and M. Enjoji, "Malignant "triton" tumors: a clinicopathologic and immunohistochemical study of nine cases," Human Pathology, vol. 15, no. 8, pp. 768-778, 1984.

[25] J. S. J. Brooks, M. Freeman, and H. T. Enterline, "Malignant 'Triton' tumors. Natural history and immunohistochemistry of nine new cases with literature review," Cancer, vol. 55, no. 11, pp. 2543-2549, 1985.

[26] S. Weiss and F. Enzinger, "Malignant tumors of peripheral nerves," in Enzinger and Weiss's Soft Tissue Tumors, S. Weiss and R. Goldblum, Eds., pp. 1209-1263, CV Mosby, St. Louis, Mo, USA, 4th edition, 2001.

[27] G. V. N. Velagaleti, M. Miettinen, and Z. Gatalica, "Malignant peripheral nerve sheath tumor with rhabdomyoblastic differentiation (malignant triton tumor) with balanced $t(7 ; 9)$ (q11.2;p24) and unbalanced translocation $\operatorname{der}(16) \mathrm{t}(1 ; 16)(\mathrm{q} 23 ; \mathrm{q} 13), "$ Cancer Genetics and Cytogenetics, vol. 149, no. 1, pp. 23-27, 2004.

[28] L. Victoria, T. McCulloch, E. Callaghan, and N. Bauman, "Malignant triton tumor of the head and neck," Head \& Neck, vol. 21, pp. 663-670, 1999.

[29] A. Capote, V. Escorial, T. Reina, M. F. Muñoz-Guerra, S. Nieto, and L. Naval, "Primary malignant schwannoma of the cervical plexus with melanocytic differentiation," International Journal of Oral and Maxillofacial Surgery, vol. 35, no. 8, pp. 767-771, 2006.
[30] A. Terzic, B. Bode, K. W. Gratz, and S. J. Stoeckli, "Prognostic factors for the malignant triton tumor of the head and neck," Head and Neck, vol. 31, no. 5, pp. 679-688, 2009.

[31] S. T. Kim, C. W. Kim, G. C. Han et al., "Malignant triton tumor of the nasal cavity," Head and Neck, vol. 23, no. 12, pp. 1075-1078, 2001.

[32] K. B. Sørensen, C. Godballe, and A. Krogdahl, "Malignant triton tumor (MTT) of the neck," Auris Nasus Larynx, vol. 33, no. 1, pp. 89-91, 2006. 


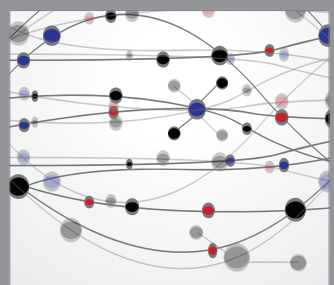

The Scientific World Journal
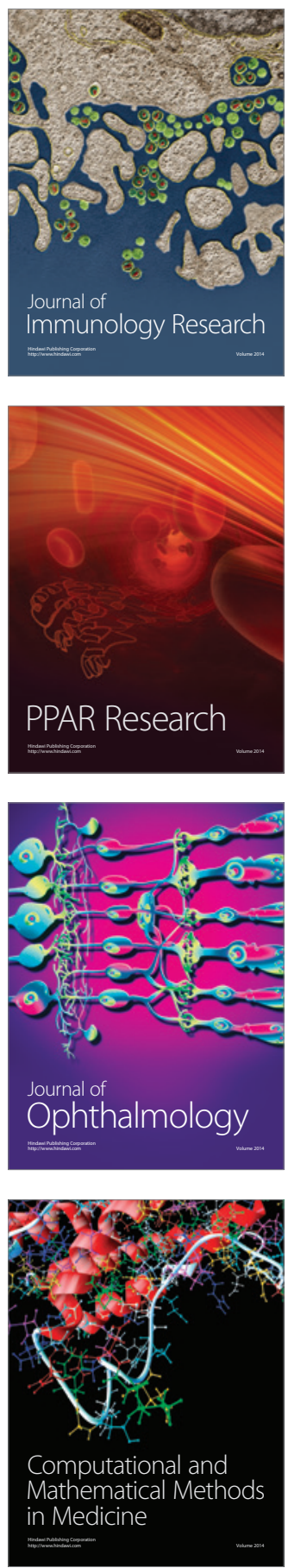

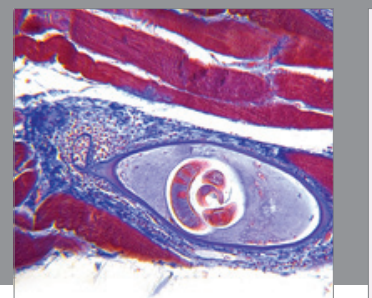

Gastroenterology

Research and Practice
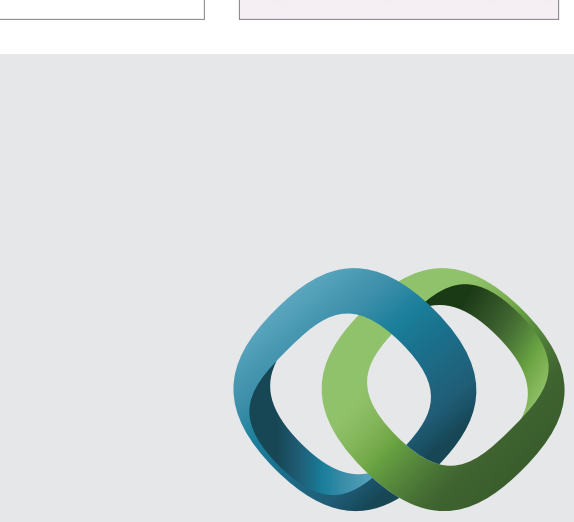

\section{Hindawi}

Submit your manuscripts at

http://www.hindawi.com
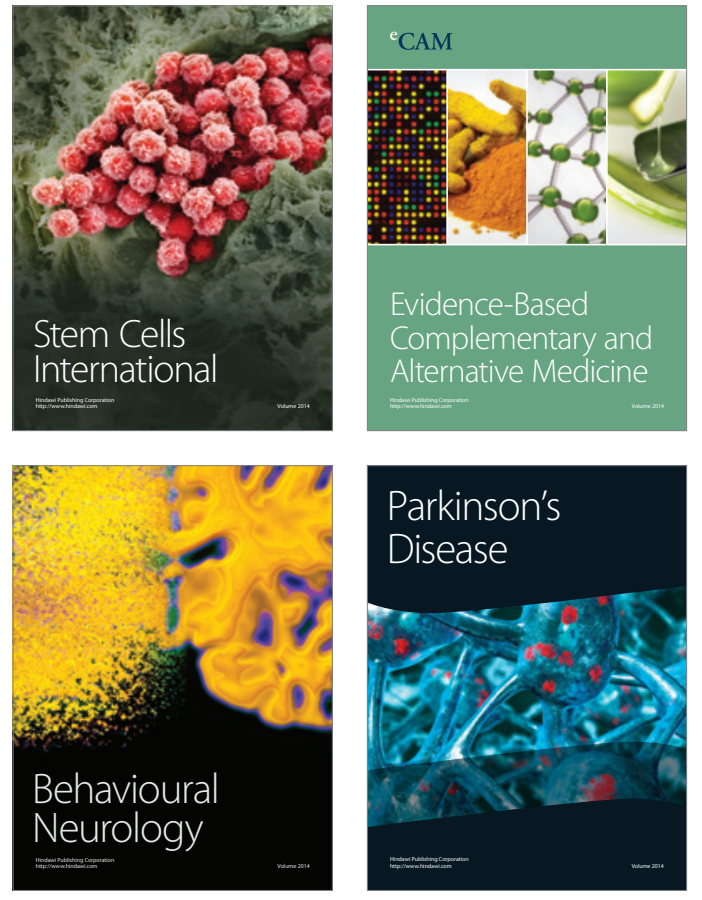
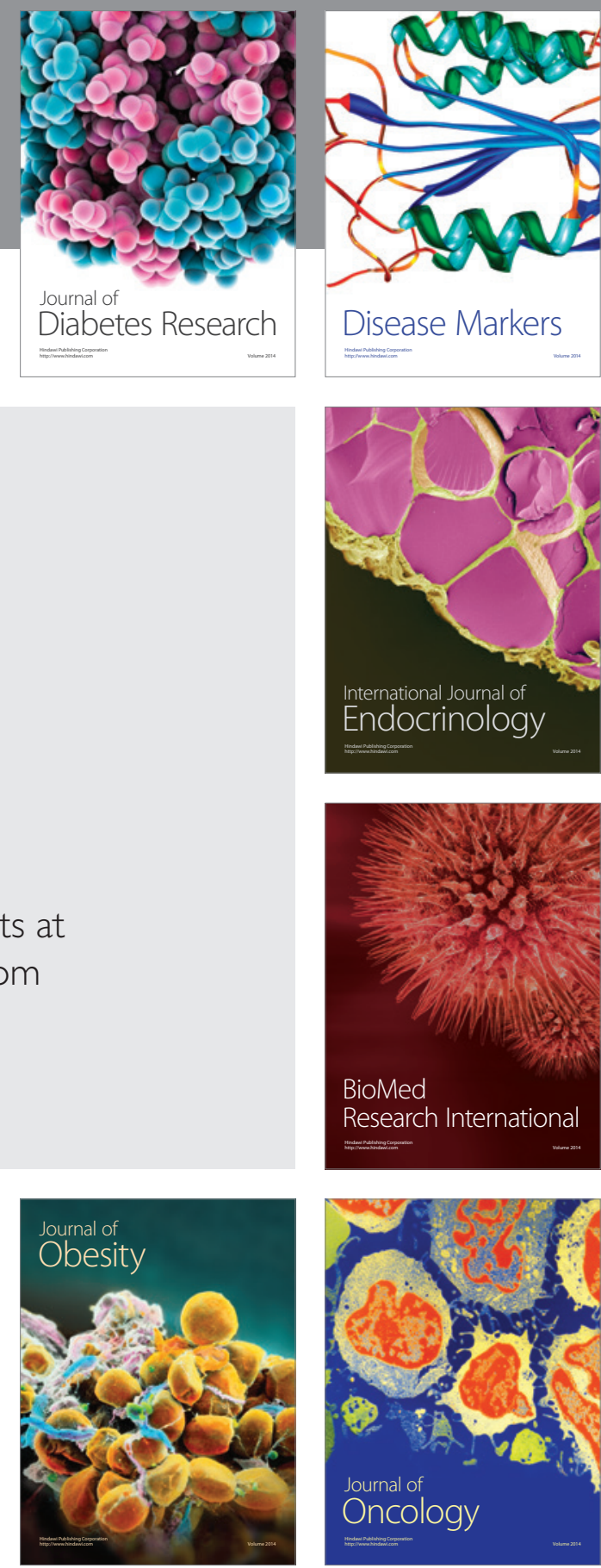

Disease Markers
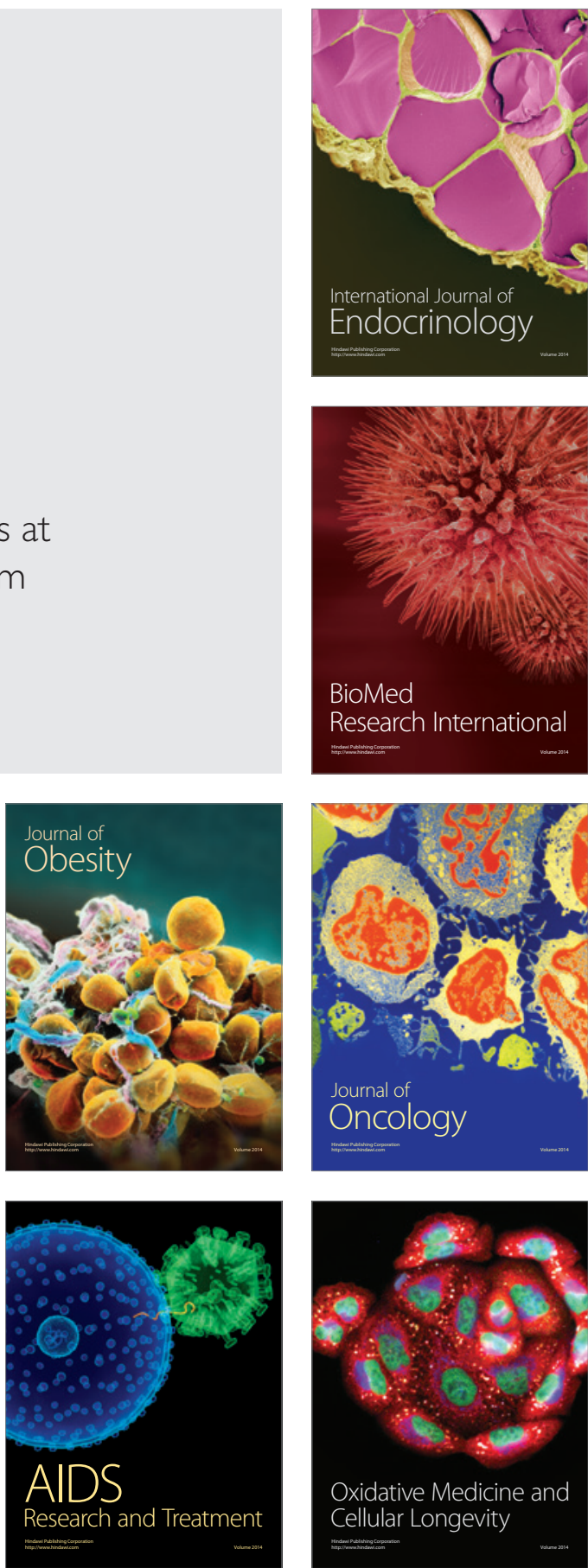